\title{
Einflüsse von Computer- und Mediennutzung auf die Persönlichkeitsentwicklung von Kindern und Jugendlichen
}

D. Süss

\section{Zusammenfassung}

Kinder und Jugendliche entwickeln ihre Persönlichkeit in aktiver Auseinandersetzung mit Medien. Medienkompetenz. ist zu einer Entwicklungsaufgabe geworden. Zugleich verändern die Medien die allgemeinen Sozialisationsprozesse wie die Integration in die Peergroup und die Ablösung von den Eltern. Medien können sowohl als Ressourcen wie alich als Risiken für die Entwicklung wirksam werden. Empirische Studien zeigen, dass Kinder einen immer reich haltigeren Medienzugang haben und viel Zeit mit Bildschirmmedien verbringen. Mediensozialisation wird zwar von den Heranwachsenden zunehmend selbst gesteuert, zur Verhinderung von negativen Medienwirkungen, zum Beispiel Internetsucht, ist aber erzieherische Aufmerksamkeit erforderlich. Wennder Medienumgang innerhalb einer kinderfreundlichen Lebenswelt statfindet, dann werden sich Medien nicht negativ auf die Entwicklung der Kinder auswirken.

\section{Theoretische Ansätze der Mediensozialisationsforschung}

Kinder und Jugendliche wachsen heute in einer Welt auf, die von Medien durchdrungen ist. Die Persön- lichkeit entwickelt sich in Auseinandersetzung mit der Mediengesellschaft. Dies wird von einer Reihe von Autoren primär aus einer pathogenetischen Perspektive betrachtet. Sie gehen davon aus, dass $\mathrm{Me}$ dien Risikofaktoren für das Aufwachsen von Kindern darstellen. «Bildschirmmedien machen dick, dumm und gewalttätig», bringt der Neurologe Manfred Spitzer [1] diese Haltung auf den Punkt. Der Kriminologe Christian Pfeiffer betont, dass der persönliche Medienbesitz der Kinder mit ihrem schulischen Erfolg oder Misserfolg korreliere. Der Medienpsychologe PeterWinterhoff-Spurk diagnostiziert, dass die Unterhaltungskultur zu «kalten Herzen» führe, und der Psychiater Michael Millner spricht den sogenannten Beta-Kindern Hyperakîivität und andere Verhaltensauffälligkeiten $\mathrm{zu}$, wobei er unter der Beta-Welt die gesamte Medienwelt versteht, vom Fernsehen bis zum Cyberspace. Schon früher wurde den Medien das Verschwinden der Kindheit oder gar das Verschwinden der Wirklichkeit angelastet, Fernsehen und Internet wurden zu Drogen erklärt und der Zerfall von Kommunikationskulturen durch Medien beklagt. Diese Ansätze sind zumeist von einer kulturpessimistischen Haltung geprägt. Die Medien werden dafür verantwortlich oder zumindest in hohem Maße mitverantwortlich gemacht, dass ein Wertezerfall stattfinde und die Grundlagen einer solidarischen und vertrauenswürdigen Gesellschaft erodierten.

Stützte sich eine solche Einschätzung früher primär auf theoretische Abhandlungen und essayistische Fallberichte, so werden heute auch großangelegte empirische Studien beigezogen, welche die vorge- 
brachten pessimistischen Thesen abzustützen scheinen. Die experimentellen Studien der Hirnforschung beeindrucken die Laien besonders stark, da hier (end lich) ein bildhafter Beweis für die einfachen intuitiven Überzeugungen vieler Erziehenden vorgelegt wird. Bei genauerem Hinsehen werden die Daten allerdings oft einseitig interpretiert, wenn beispielsweise das Medienverhalten von Migrantenkindern mit demjenigen von anderen Kindern verglichen wird, ohne zu berücksichtigen, dass man damit die konfundierte Variable des sozialen Milieus, der finanziellen Möglichkeiten und des Bildungshintergrundes mit im Spiel hat.

Die Rolle der Medien wird von einer anderen Autorengruppe eher unter salutogenetischer Perspektive betrachtet. Medien können Entwicklungsanregungen bieten. Sie können auch Anregungsdefizite in der realen Umwelt, der Alpha-Welt nach Millner, kompensieren. Sie sind protektive Faktoren in einer Welt, die hohe Ansprüche an die Kinder stellt. In dieser Gruppe, die von mehr oder weniger kritischem Optimismus in Bezug auf die Sozialisationsfunktion von Medien geprägt ist, wird betont, dass ein Auf wachsen in einer Mediengesellschaft dann optimal verläuft, wenn das Kind die Angebote der Medien früh zu nutzen beginnt und so zu einem kompetenten und spielerischen Medienumgang findet. Kommunikations- und Medienkompetenz sind nach dem Erziehungswissenschafter Dieter Baacke die Grundlagen der gesellschaftlichen Partizipation. Der Medienumgang wird zur Kulturtechnik erklärt. Alphabetisierung umfasst heute eine Medienalphabetisierung, betont etwa der Medienpädagoge Christian Doelker [2]. Die neuen Kommunikationskulturen werden nicht einfach als defizitär gegenüber früheren Formen beschrieben, sondern die Neuerungen werden neutral bis begeistert als kulturelle Weiterentwicklung verstanden, an der die Kinder nicht nur teilhaben, sondern welche die Kinder als Innovatoren der Gesellschaft sogar erst hervorbringen.

Eine Verbindung zwischen der pathogenetischen und der salutogenetischen Denkrichtung besteht darin, dass die Medien sowohl als Risiken als auch als Ressourcen für die Persönlichkeitsentwicklung der Kinder verstanden werden. Medienerziehung und Medienbildung sind dann darauf ausgerichtet, die Risikopotenziale ohne polemische Rhetorik zu erfassen und die Ressourcen, welche die Medien darstellen können, ohne euphorische Idealisierungen zu benennen.

\section{Sozialisation als Teil der Persönlichkeitsentwicklung}

Die Persönlichkeitsentwicklung von Sozialisanden (Individuen, welche in eine Gemeinschaft hineinwachsen) vollzieht sich in aktiver Auseinandersetzung mit Sozialisationsinstanzen und Sozialisations- akteuren. Instanzen wie Familie, Schule, Berufsbildung und Kirchen haben einen expliziten Sozialisationsauf trag. Sie wollen und sollen neue Mitglieder in ihre Gemeinschaften integrieren, ihnen die Regeln vermitteln, welche es zu beachten gilt, wenn man vom Novizen zum vollwertigen Mitglied einer Gemeinschaft werden will. Handlungsfreiräume werden schrittweise vergrößert. Die Sozialiationsagenten (z.B. Medien, Peergroups, Fan-Gemeinschaften und Sportvereine) haben keinen expliziten Sozialisationsauftrag, nehmen aber dennoch eine sozialisierende Funktion wahr, oft sogar in höherem Maße als die dazu bestimmten Instanzen. Das Ziel der Sozialisation besteht darin, ein gesellschaftlich handlungsfähiges Subjekt zu werden. Die gesellschaftliche Bedingtheit von individuellen Entwicklungsprozessen wird in der Sozialisationsforschung untersucht, die Entwicklung von Handlungskompetenzen erklärt, soziale Gruppen auf der Grundlage von geteilten Bedeutungen analysiert. Die Persönlichkeitsentwicklung wird als Aufbau von Sinnentwürfen verstanden in einer Multioptionsgesellschaft, welche eine Vielzahl an gegenseitigen Relativierungen von Lebensstilen umfasst.

Ältere Sozialisationstheorien verstanden Sozialisation primär als Anpassung des Individuums an die soziale Umgebung. Persönlichkeitsentwicklung wurde exogenistisch begründet. Neuere Ansätze verstehen Sozialisation als aktive Auseinandersetzung mit Rollendefinitionen. Gesellschaftliche Rollen werden vom Sozialisanden nicht einfach übernommen, sondern kritisch geprüft und weiter entwickelt. Das Individuum wählt jene sozialökologischen Felder aus, in denen die persönlichen Voraussetzungen und die sozialen Erwartungen möglichst gut zur Passung kommen können. Es bestehen weiterhin Entwicklungsbereiche mit klar definierten normativen Vorgaben (z.B. Altersnormen für den «richtigen» Zeitpunkt der Einschulung), wo Fremdsozialisation vorherrscht. Das Feld der Selbstsozialisation nimmt aber an Bedeutung zu, d.h. die Sozialisationsinstanzen lassen den Sozialisanden viele Optionen offen, welche gleich hoch gewertet werden. Die Kinder als Sozialisanden entwickeln sich selbstgesteuert, respektive sie lernen von ihren Peers. Dies ist gerade im Bereich des Medienumgangs bedeutsam. Sozialisanden wirken schließlich auf ihre Sozialisatoren zurück. Diese sogenannte reziproke Sozialisation erfahren Eltern und Lehrpersonen gerade auch im Zusammenhang mit dem Medienalltag der Heranwachsenden.

Die Grundfragen der Mediensozialisationsforschung lassen sich zusammenfassend in zwei Bereiche gliedern: 1) Sozialisation zur kompetenten Medienkommunikation. 2) Gelingende Sozialisation durch Medienkommunikation. Es gilt zu fragen, wie Menschen den Umgang mit Medien lernen und welche Formen des Medienumgangs sich unterscheiden lassen. Der 
kompetente Medienumgang ist zu einer Entwicklungsaufgabe geworden. Die Medien verändern aber auch die allgemeinen Sozialisationsprozesse. Hier ist wichtig zu erfassen, welche entwicklungsfördernden oder -gefährdenden Veränderungen durch die Medialisierung der Gesellschaft festzustellen sind.

\section{Mediensozialisation, Erwerb von Kompetenzen und Identität}

Un die Rolle der Medien für die Persönlichkeitsentwicklung zu klären und damit die Mediensozialisation zu beschreiben, sollen im Folgenden ein paarausgewählte Forschungsbefunde vorgestellt werden. Sie umfassen die Mediennutzung mit den Ausprägungen des Zugangs, der Erwartungen, Funktionen und Bindungen. Medienrezeptionsstile und Medienwirkungen leiten sich daraus ab. Die hier diskutierten schweizerischen Studien umfassen den Zeitraum 1996 bis 2005, greifen aber auch auf frühere Studien bis in die fruihen 1970er-Jahre und auf den internationalen Vergleich zurück [3-7].

Von den 70er- zu den 90er-Jahren konnte für die Schweiz gezeigt werden, dass die Medienausstattung der Kinderzimmer erheblich zugenommen hat. Bücher und auditive Medien waren schon früher wichtig, sie kommen heute aber auch schon bei jüngeren Kindern vor. Je älter die Kinder sind, desto vielfältiger ist ihr Medienensemble (Tab. 1 und 2).

Tabelle 1 Medien im Kinderzimmer (Schweiz, 1976) In Prozent der Befragten (nach Altersgruppen, $N=2759$ )

\begin{tabular}{lccc}
\hline Medium & 8-10 Jahre & 11-13 Jahre & 14-16 Jahre \\
\hline Freizeit-Bucher & 94 & 96 & 95 \\
Radio & 41 & 65 & 82 \\
Comics & 75 & 70 & 41 \\
Zeitschriften & 42 & 56 & 62 \\
Plattenspieler & 42 & 68 & 85 \\
Fernseher & 7 & 4 & 9 \\
\hline
\end{tabular}

Tabelle 2 Medien im Kinderzimmer (Schweiz, 1996) In Prozent der Befragten (nach Altersgruppen, $N=764$ )

\begin{tabular}{lccc}
\hline Medium & 8-10 Jahre & 11-13 Jahre & 14-16 Jahre \\
\hline Freizeit-Bücher & 95 & 98 & 93 \\
Radio & 81 & 89 & 92 \\
Comics & 69 & 77 & 62 \\
Zeitschriften & 37 & 70 & 69 \\
Stereoanlage & 33 & 67 & 80 \\
Fernseher & 12 & 18 & 26 \\
Gameboy & 50 & 50 & 46 \\
Walkman & 49 & 76 & 80 \\
Computer & 12 & 20 & 27 \\
Spielkonsole & 12 & 15 & 16 \\
Zeitungen & 7 & 11 & 21 \\
Videoplayer & 7 & 6 & 8 \\
Internet & 3 & 2 & 3 \\
\hline
\end{tabular}

Tabelle 3 Medien im Zimmer von 10- bis 18-Jăhrigen (Schweiz, 2005)

In Prozent der Befragten (nach Geschlecht, $N=1630$ )

\begin{tabular}{lccc}
\hline Medium & Mädchen & Knaben & Alle \\
\hline Musikanlage & 93 & 90 & 91 \\
Freizeit-Bücher & 94 & 87 & 91 \\
Radio & 90 & 87 & 88 \\
Walkman & 82 & 70 & 76 \\
Handy & 76 & 69 & 72 \\
Gameboy & 48 & 61 & 55 \\
Moderner PC & 38 & 47 & 43 \\
MP3-Player & 30 & 45 & 38 \\
Fernseher & 23 & 35 & 29 \\
Internet & 26 & 31 & 28 \\
Video-/DVD-Player & 19 & 31 & 25 \\
Spielkonsole & 13 & 35 & 24 \\
Kabel/Satelliten-TV & 19 & 24 & 21 \\
Teletext & 18 & 24 & 21 \\
DVD-Recorder & 11 & 20 & 16 \\
Videokamera & 12 & 17 & 14 \\
Telefon (Festnetz) & 14 & 13 & 13 \\
Älerer PC & 7 & 8 & 7 \\
\hline
\end{tabular}

Eine Studie von 2005 zeigte, dass insbesondere Handy, Internet und MP3-Player als neue Medien die Kinderzimmer rasch erobert haben, dass die Geschlechterunterschiede im Medienzugang aber nach wie vor auffällig sind. Mädchen haben sich das Handy schneller angeeignet als die Jungen. Sie telefonieren und senden SMS lieber und öfter als die Jungen. Sie lesen auch nach wie vor mehr in Büchern. Die Jungen besitzen deutlich mehr Computermedien, sie spielen insbesondere ausgiebiger und leidenschaftlicher am Computer als die Mädchen. Sie spielen auch anderes: Sind es bei den Jungen Action, Abenteuer und Sportspiele, so sind es bei den Mädchen die Spiele auf dem Handy oder die interaktive Soap-Opera «die Sims», welche zu den Favoriten zăhlen (Tab. 3).

Die elterliche Kontrolle des Medienumgangs hat sich in den letzten Jahrzehnten wenig verändert, wie ein Vergleich mehrerer Erhebungen aus der Schweiz zeigt (Tab. 4). Befragt danach, welche Medien regelmäßig zu Konflikten in der Familie führen, steht nach wie vor das Fernsehen an erster Stelle, besonders wenn das Kind kein eigenes Gerät besitzt und in der Familie der «Kampf um die Fernbedienung» ausgefochten wird. Dieser wird meist vom Vater gewonnen. Aber auch das Handy und die Onlinespiele sind zu Konfliktfeldern geworden. $10 \%$ von befragten Deutschschweizer 10- bis 18-Jährigen sagten im 2005 von sich, Mitglied eines Onlinespiel-Clans zu sein. Dabei werden oft ganze Nächte durchgespielt, zusammen mit einem Team, das international zusammengesetzt sein kann.

Oft sind es aber auch Freunde aus der Schule oder Nachbarschaft, die zusammen online spielen oder sich zu LAN-Parties treffen. Insbesondere bei Ju- 
Tabelle 4 Elterliche Kontrolle der Fernsehnutzung in der Schweiz (Vier Erhebungen des SRG Forschungsdienstes, 5- bis 14-jährige Kinder)

\begin{tabular}{lcccc}
\hline $\begin{array}{l}\text { Angaben der Kinder } \\
\text { (in Prozent) }\end{array}$ & 1971 & 1979 & 1983 & 1995 \\
\hline Darf alles sehen & 19 & 12 & 17 & 17 \\
Muss manchmal fragen & 37 & 48 & 45 & 39 \\
Muss immer fragen & 44 & 35 & 38 & 42 \\
Darf nie fernsehen & 0 & 0 & 0 & 2 \\
\hline Anzahl Befragte N $=$ & 2483 & 2396 & 500 & 2003 \\
\hline
\end{tabular}

gendlichen unter 20 Jahren kann die Internetnutzung ein Suchtpotenzial beinhalten. Vor allem Spiele, Chatforen und pornografische Inhalte können ein unkontrolliertes Medienverhalten fördern: Die Nutzungszeiten nehmen zu (20-35 Stunden pro Woche), es entsteht ein unwiderstehlicher Drang, online zu gehen, das Surfen wird verheimlicht. Arbeit, Ernährung, Erholung und Hygiene werden vernachlässigt. Solche Alarmsignale sollten ernst genommen werden. Suchtberatungsstellen verfügen bereits über spezialisierte Hilfsangebote [8-9].

Das Handy ist nicht nur zur «verlängerten Leine» für die Heranwachsenden geworden, sondern es ermöglicht auch den Eltern einen mobileren und flexibleren Lebensstil. Via Handy bleiben sie für die Kinder erreichbar, nicht nur, wenn diese im Ausgang sind, sondern auch wenn die Eltern beruflich oder freizeitlich unterwegs sind. Die Jugendlichen erleben das Handy auch als Medium des Flirts und des Probehandelns. Sie setzen dieses und andere Medien dazu ein, Entwicklungsaufgaben zu bewältigen, wie der Unbau der sozialen Beziehungen in der Pubertät, der Umgang mit Intimität und Sexualität und der Umgang mit dem Warenmarkt. Die Markenbindung der Jugendlichen wird als Mittel der subkulturellen Positionierung genutzt. Wer nicht die richtige Handymarke besitzt, kann ausgegrenzt werden.

In einer explorativen Befragung der Hochschule für Angewandte Psychologie (HAP) von 14- bis 16-Jährigen im Jahr 2004 konnte festgestellt werden, dass der Computer bei manchen Jungen zum wichtigsten persönlichen Objekt geworden ist. Hütete ein Kind früher seine wichtigsten Schätze in einer Schuhschachtel, so sind es heute Handy und Computer, welche diese Rolle übernommen haben: Digitale Fotos, SMS, Adressen von Freundinnen und Freunden, Bilder von Stars, heruntergeladene Musik und Filmchen gehören zur virtuellen geheimen Schatzkammer der heutigen Heranwachsenden. Audiovisuelle Medien und Bücher erreichen nicht diese Schlüsselrolle (Tab. 6).

Im Zusammenhang mit Medienwirkungen wird oft beklagt, dass die Kinder immer asozialer und materialistischer würden. Wir fragten daher die Kinder
Tabelle 5 Welche Eigenschaften machen jemanden in deinem Alter beliebt? (11 - bis 16-jäbrige Befragte, Schweiz 1997)

\begin{tabular}{lcc}
\hline $\begin{array}{l}\text { Eigenschaft, um beliebt zu sein } \\
\text { (in Prozent der Befragten) }\end{array}$ & $\begin{array}{c}\text { Mädchen } \\
(\mathrm{N}=546)\end{array}$ & $\begin{array}{c}\text { Jungen } \\
(\mathrm{N}=38 \mathrm{l})\end{array}$ \\
\hline Hilfreich/freundlich sein & 58 & 52 \\
Ehrlich sein & 47 & 43 \\
Natürlich/sich selber sein & 46 & 32 \\
Humorvoll sein & 39 & 44 \\
Gut aussehen & 35 & 33 \\
Die nchtigen Kleider tragen & 31 & 36 \\
Gut in der Schule sein & 16 & 19 \\
Originell/anders & & \\
als die anderen sein & 16 & 17 \\
Die neusten Sachen haben & 13 & 15 \\
Gut im Sport sein & 9 & 22 \\
Geld ausgeben können & 7 & 15 \\
\hline
\end{tabular}

Tabelle 6 Schaffi Besitz Identität?

Die Rolle persönlicher Objekte für Jugendliche (14- bis 16-jährige Befragte, Schweiz 2004: HAP, Matthias Maurer)

\begin{tabular}{lcc}
\hline $\begin{array}{l}\text { Personliche Objekte } \\
\text { (in Prozent der Befragten) }\end{array}$ & $\begin{array}{c}\text { Mädchen } \\
(\mathrm{N}=30)\end{array}$ & $\begin{array}{c}\text { Jungen } \\
(\mathrm{N}=30)\end{array}$ \\
\hline Haustier & 47 & 43 \\
Computer & 20 & 63 \\
Fotos & 40 & 40 \\
Handy & 40 & 27 \\
Spornartikel & 17 & 40 \\
Plischtier & 33 & 10 \\
Kleider & 27 & 3 \\
Audiovisuelle Medien & 13 & 10 \\
Sammelobjekte & 3 & 17 \\
Bücher & 10 & 3 \\
Musikinstrument & 7 & 3 \\
\hline
\end{tabular}

nach ihren Werthaltungen. Auf die Frage hin, was jemanden in ihrem Alter unter den Peers beliebt mache, nannten die 11-bis 16-Jährigen meist an erster Stelle prosoziale Werte: Man muss freundlich, humorvoll, natürlich und ehrlich sein. Die Jungen nannten doppelt so oft als Mädchen, dass man auch viel Geld ausgeben können muss (Tab. 5). Auch im Hinblick auf die Frage, was für sie wichtig sein werde, wenn sie erwachsen sind, nannten Jungen doppelt so oft wie Mädchen, dass sie über viel Geld verfügen wollen. Aber auch dann überwiegten die Wünsche nach guten Freunden, einer eigenen Familie und einem interessanten Beruf. Diese Schweizer Befunde decken sich mit Studien von Jürgen Zinnecker in Deutschland.

Wie stellen nun Jugendliche ihre Persönlichkeit dar? Waren es früher Poesiealben, Zuschriften an Jugendzeitschriften, die Selbstinszenierung durch Frisuren, Bekleidung und weitere Accessoires, so sind es heute (zusätzlich) Selbstinszenierungen im Internet. 
Blogs und Online-Portale wie www.myspace.com sind zur virtuellen Probebühne der Selbstdarstellung von Teenagern und Adoleszenten geworden. Wir haben die schweizerische Plattform www.meinbild.ch näher analysiert. Die Jugendlichen stellen ihre Portraitfotos ins Netz, sie schreiben Gedichte, benennen ihre Stars und Leidenschaften. Sie stellen ihre Freunde, Haustiere und Hobbies vor - und sie suchen auf dem schnellen Weg via E-Mail nach Kontakten. Manche Eltern sind besorgt oder schockiert über die Freizügigkeit mancher Selbstinszenierungen. Die Jugendlichen präsentieren sich auf den Fotos manchmal auch in verführerischen Posen und Blicken. Allerdings betonen Jugendliche in Interviews, dass sie im Alltag dann viel zurückhaltender seien. Sie nutzen die virtuelle Umgebung als Testgebiet, aus dem sie sich jederzeit auch wieder ausklinken können. Dass damit auch Gefahren verbunden sein können, von Mobbing durch andere bis zu sexueller Belästigung, muss den Jugendlichen dabei durch die Erziehenden kritisch vor Augen geführt werden. Manche Jugendlichen betonen aber auch, das Ganze sei nicht ernst gemeint, sondern ein Spaß, wie das Ausschmücken eines Avatars (virtueller Stellvertreter, Spielfigur, die man steuert) mit Attributen der eigenen WunschIdentität.

\section{Online-Beratung als attraktives Angebot für die Net Kids}

Dass sich die Erfahrungen der Jugendlichen mit virtueller Kommunikation auch auf ernsthafte Kommu- nikationsanlässe mit Erwachsenen überträgt, zeigt eine Studie der Hochschule für Angewandte Psychologie (HAP) von 2005 zur Frage, wie Jugendliche Onlineberatung in Anspruch nehmen. Gemäß einer Befragung von 15- bis 22-Jährigen in der Deutschschweiz waren $29 \%$ schon auf Online-Beratungsangebote gestoßen und $24 \%$ haben sie auch genutzt (19\% der Jungen und 28\% der Mädchen). Das wichtigste Thema, zu dem Onlineberatung beansprucht wurde, war Schule/Arbeit, gefolgt von Suchtproblemen. Andere Themen, wie Sexualität, Familie und Beziehungen spielten nur eine untergeordnete Rolle. Die Jugendlichen betonten, dass sie es schätzen, nicht viel von ihrer Identität preisgeben zu müssen bei dieser Form der Beratung und rasch und unkompliziert Beratung zu erhalten. Zugleich legen sie Wert darauf, möglichst viel Informationen über den Berater oder die Beraterin zu erhalten. Es wird also eine asymmetrische Kommunikationssituation geschaffen, in der die Jugendlichen sich wohl fühlen, da sie über ein hohes $\mathrm{Maß}$ an Kontrolle über das Ausmaß ihrer Selbstkundgabe verfügen.

\section{Medienkonsum einschränken?}

Die Mediennutzung von Kindem und Jugendlichen führt weder dazu, dass eine Mehrheit «dick, dumm und gewalttätig oder traurig» wird, noch ist es in jedem Fall eine Bereicherung, wenn alle neuen Medien möglichst schnell im Kinder- und Schulzimmer landen. Je nach Passung zwischen Medienangeboten und den Entwicklungsthemen und -aufgaben der He-

Tabelle 7 Empfehlungen zum Umgang mit Medien

Empfehlung für maximale tägliche Freizeit vor dem Bildschirm (TV und Computer)

- Kleinkinder: keine Bildschirmmedien

- Vorschulkinder: 30 Minuten

- Unterstufenalter: 30-60 Minuten

- Mittelstufenalter: 60-90 Minuten

- Oberstufenalter: 90-120 Minuten

Gut gewählter Standort der Medien

- Fernseher nicht so im Wohnzimmer platzieren, dass er zum Zentrum wird

- Kein Fernseher im Kinderzimmer

- Bücher und Musilmedien so platzieren, dass sie einladend wirken

Medienkompetenz fördern

- Die Kinder in der Auswahl geeigneter Filme, Spiele, Websites und Bücher beraten

- Kinderschutz fürs Internet vorsehen: Filtersoftware und den Kindern Verhaltensregeln erlăutern (z.B. nie die eigene Adresse angeben): vgl. www.klicksafe.de

- Unerwünschte Medieninhalte benennen und erklären, weshalb man sie ablehnt

Medien in einem anregenden Alltag integrieren

- Immer wieder Tage ohne Bildschirmmedien vorsehen

- Bewegung und gesunde Ernähnung fördem

- Über positive und negative Medienerfahrungen miteinander reden

- Darauf achten, dass Medien nicht als Fluchtmittel verwendet werden bei sozialer Isolation oder Leistungsproblemen in der Schule 
ranwachsenden, und je nach den verfügbaren Medienkompetenzen, werden die Effiekte des Medienumgangs unterschiedlich ausfallen. Das ist nicht gerade eine einfache Ausgangslage für Eltern, Lehrende, KinderärztInnen oder -psychologInnen, welche nach möglichst allgemeingültigen Empfehlungen für dje Medienerziehung suchen. Alle medienpädagogischen Ratschläge, wie in der obigen Tabelle 7, müssen daher mit dem Hinweis versehen werden, dass die besonderen Merkmale des Kindes und seines Umfeldes einbezogen werden müssen.

Das selbstständige, aber zugleich verantwortungsvolle Medienhandeln wird immer früher in der Biographie der Kinder zu einer Entwicklungsaufgabe. Im Handling von Computer und Mobiltelefon sind die Heranwachsenden den Erwachsenen oft weit überlegen. Aber zur Reflexion der Medienwirkungen und zur Auswahl geeigneter, unterhaltsamer und lehneicher Medieninhalte werden sie immer den erzieherischen Dialog mit den Erwachsenen benötigen. So können Kinder zum Beispiel nicht abschätzen, dass zu wenig Bewegung wegen langer sitzender Haltung vor Bildschirmmedien, begleitet von selbstvergessenem Junkfood-Konsum, Adipositas begünstigen kann.

Die jungen Sozialisanden haben auch ein Recht darauf, dass Erwachsene ihnen unter aufmerksamer Begleitung Zugang zu anregenden Medienangeboten geben (Beta-Welt) und sie dazu befähigen, sich den Zugang und die kritische Bewertung im Laufe des Älterwerdens zunehmend selbst zu erschließen. Zugleich haben die Kinder ein Recht auf eine anregende Alpha-Welt, d.h. auf direkte Erfahrungen mit Natur und Mitmenschen in einer kinderfreundlichen Lebenswelt.

\section{Literatur}

1. Spitzer M. Vorsicht Bildschirm! Elektronische Medien, Gehirnentwicklung, Gesundheit und Gesellschaft. Stuttgart: Ernst Klett, 2005.

2. Doelker C. KuJturtechnik Fernsehen. Analyse eines Mediums. Stuttgart: Klett-Cotta, 1989.

3. Süss D, Schlienger A, Kunz Heim D, Basler M, Böhi S, Frischknecht D. Jugendliche und Medien. Merkmale des Medienalltags, unter besonderer Berücksichtigung der Mobilkommunikation. Zürich: Hochschule für Angewandte Psychologie, 2003a. Online: http://www.hapzh.ch/ download/F_Jugendliche_und_Medien.pdf
4. Süss D, Rutschmann V, Böhi S, Merz C, Basler M, Mosele F. Medienkompetenz in der Informationsgesellschaft. Selbsteinschätzungen und Ansprüche von Kindern, Eltern und Lehrpersonen im Vergleich. Zürich: Hochschule für Angewandte Psychologie, 2003b. Online: http://www. hapzh.ch/download/F_Medienkompetenz_153.pdf

5. Süss D. Mediensozialisation von Heranwachsenden. Dimensionen, Konstanten, Wandel. Wiesbaden: VS Verlag für Sozialwissenschaften, 2004.

6. Süss D, Marti Salzmann G. Medien im Alltag von Schülerinnen und Schülern. Zürich: Hochschule für Angewandte Psychologie, 2006. Online: http://www.hapzh.ch/ download/F_KMMedienImAlItag2006-07.pdf

7. Livingstone $S$, Bovill $M$. eds. Children and their changing medja environment. A European comparative study. Mahwah, NJ. London: Lawrence Eribaum Associates, 2001.

8. Grüsser $S$, Thalemann C. Verhaltenssucht. Diagnostik, Therapie, Forschung. Bern: Hans Huber, 2006.

9. Offene Tür Zürich. Abhängig von der virtuellen Welt. 2006. Online: http://www.offenetuer-zh.ch/onlinesucht. html

\section{Summary: \\ Impacts of computer- and media usage on the personality develop- ment of children and young people}

The psychosocial development of children and the youth today is embedded in a media society. Socialization is understood as an interaction between the individual and its environment. Media are used to accomplish developmental tasks and media literacy has become a developmental task in itself. The presence of media in all social subsystems of every day life alters the general socialization processes, like the integration into peergroups or the detaching from the parents. Media can play the role of resources or the role of risk factors for the development. Empirical research shows that children's access to media is more and more enhanced and an increasing amount of time is spent with screen media. Media socialization of the young pe ple takes on the mode of self education, but children are dependent on adults to prevent negative media effects such as Internet addiction. If media usage is part of an environment which is adequat for children's wellbeeing, the psychosocial development will not be affected negatively by the media. 